\title{
Präzedenzfall: Langzeitphysiotherapie in Alters- und Pflegeheimen
}

Beat Kunz

* Tarif über die Vergütung
physiotherapeutischer Leistungen.

Korrespondenz:

Beat Kunz

Schweizer Physiotherapie Verband

Stadthof

Bahnhofstrasse $7 \mathrm{~b}$

CH-6210 Sursee

Tel. 0419260780

Fax 0419260799

info@fisio.org

www.fisio.org
Eine Krankenversicherung wollte in einem Grundsatzentscheid erwirken, dass physiotherapeutische Leistungen in Alters- und Pflegeheimen mit Hinweis auf die fehlende Wirksamkeit nicht mehr durch Physiotherapeuten, sondern durch das Pflegepersonal auszuführen sind. Das Gericht entschied gegen die Krankenkasse.

Die Krankenversicherung behauptete, dass die täglichen Bewegungsübungen des Pflegepersonals eines Pflegeheimes wirksamer seien als die - ärztlich verordnete - physiotherapeutische Behandlung nach Bobath und diese deshalb im konkreten Fall nicht medizinisch indiziert sei. Das Verwaltungsgericht des Kantons Thurgau hat die Klage der Krankenversicherung gegen einen Physiotherapeuten abgewiesen.

\section{Gericht stützt physiotherapeutische Behandlung}

In seinen Erwägungen grenzte das Gericht die Grundpflege nach Art. 7 Abs. 2 lit. c Ziff. 1 KLV zu der auch Bewegungsübungen, Mobilisieren und Dekubitusprophylaxe gehören - von einer physiotherapeutischen Behandlung nach Bobath (Art. 5 Abs. 1 lit. h Ziff. 3 KLV) ab. Es hält fest, dass im Rahmen des Bobathkonzepts tägliche Bewegungsübungen durch das Pflegepersonal nötig sind und zur Grundpflege gehören, diese in aller Regel jedoch nur «en passant» und nicht therapeutisch gezielt vermittelt werden. Im Gegensatz dazu werde in der Physiotherapie eine wissenschaftlich anerkannte Therapiemethode angewandt, die zur Zustandserhaltung und Risikoverminderung eingesetzt werde (Erwägung 2e). Es kam zum Schluss, dass die Grundpflege allein den Zustand der Patientin nicht zu halten beziehungsweise zu verbessern vermag. Somit ist die physiotherapeutische Behandlung nach Bobath sowohl als wirksam (da wissenschaftlich anerkannt) als auch als wirtschaftlich (da nicht durch die Grundpflege abgedeckt) anzusehen.

Ebenso folgte das Gericht der Praxis der Paritätischen Vertrauenskommission PVK, 42 Sitzungen pro Jahr mit der Tarifposition 7311* als grundsätzlich wirtschaftlich anzusehen unter dem Vorbehalt einer nach oben abweichenden ärztlichen Anordnung aufgrund der besonderen Situation der Versicherten.

\section{Fazit}

Mit dem vorliegenden Grundsatzentscheid des Verwaltungsgerichtes des Kantons Thurgau erhalten Patienten verordnete Physiotherapie auch in Alters- und Pflegeheimen durch diplomierte Physiotherapeuten. Verweigert eine Krankenversicherung die Kostenübernahme von physiotherapeutischen Leistungen mit der Begründung, diese seien durch das Pflegepersonal vorzunehmen, verstösst dies gegen das oben beschriebene Urteil.

Dieser Gerichtsfall zeigt auf, dass es sich lohnen kann, bei Differenzen mit den Krankenversicherungen nicht klein beizugeben. 